\title{
Os inibidores de tirosino quinase de segunda geração
}

\section{The inhibitors of tyrosine kinase}

Márcia T. Delamain ${ }^{1}$

Mônika Conchon ${ }^{2}$
O imatinibe tem sido confirmado como terapia de primeira linha para a Leucemia Mielóide Crônica (LMC) por apresentar respostas duradouras na maior parte dos pacientes, principalmente nos que se encontram em fase precoce da doença. Entretanto, resistência ou intolerância ao imatinibe podem ocorrer. A resistência ao imatinibe ocorre com muito mais freqüência em fases mais avançadas da doença, sendo a causa mais comum o desenvolvimento de mutações no sítio BCR-ABL. Em face deste problema, novos inibidores de tirosino quinase têm sido desenvolvidos, com maior potência, diminuindo assim a chance de desenvolvimento de resistência ao tratamento. O nilotinibe e o dasatinibe são dois exemplos de inibidores de segunda geração de tirosino quinase recentemente aprovados. Ambos têm demonstrado excelentes resultados em pacientes que desenvolvem resistência ou são intolerantes ao imatinibe. Rev. bras. hematol. hemoter. 2008; 30(Supl.1):37-40.

Palavras-chave: Inibidor de tirosino quiinase; AMN107; leucemia mielóide crônica; resistencia ao imatinibe; dasatinibe.

\section{Introdução}

Enquanto o imatinibe é efetivo na maior parte dos pacientes com LMC, alguns ainda em fase crônica e uma maior proporção em fases mais avançadas são resistentes ou intolerantes ao imatinibe. ${ }^{1}$ A resistência ao imatinibe é incomum em pacientes em fase crônica (FC) inicial, enquanto a incidência estimada de resistência em dois anos é de 10\%-20\% em LMC -FC após falha ao interferon- $\alpha, 40 \%-50 \%$ em fases acelerada e $70 \%-80 \%$ em crise blástica ou leucemia linfóide aguda (LLA) $\mathrm{Ph}+{ }^{2}$

Alguns pacientes falham ao tratamento inicialmente (resistência primária), enquanto outros perdem uma resposta previamente adquirida (resistência secundária), sendo esta última a mais comum e associada ao desenvolvimento de mutações no sítio BCR-ABL. ${ }^{3,4}$ As opções terapêuticas para pacientes resistentes ou intolerantes ao imatinibe são limitadas. Inibidores de tirosino quinase de segunda geração fo- ram desenvolvidos com mais potência do que o imatinibe, com a finalidade de diminuir a chance de desenvolvimento de resistência. ${ }^{2}$

\section{Nilotinibe}

O nilotinibe é uma nova aminopiridina, disponível na forma oral, que é um inibidor ATP-competitivo da atividade da proteína tirosino quinase do BCR-ABL, prevenindo a ativação das vias mitogênico e antiapoptótica dependentes do $\mathrm{BCR}-\mathrm{ABL}$, levando à morte do fenótipo do BCR-ABL. ${ }^{4,5} \mathrm{Da}$ dos de estudos pré-clínicos demonstram que o nilotinibe atinge concentrações intracelulares mais elevadas do que o imatinibe e inibe a atividade da tirosino quinase do BCR$\mathrm{ABL}$ induzindo a apoptose em concentrações mais baixas do que o imatinibe. ${ }^{6-7}$

Estudos de fase I em 119 pacientes com LMC que foram resistentes ou intolerantes ao imatinibe, em todas as fases da doença e pacientes com LLA $\mathrm{Ph}+{ }^{6}$ mostraram resulta-

${ }^{l}$ Médica Assistente da Disciplina de Hematologia e do Centro de Hematologia e Hemoterapia da Faculdade de Ciências Médicas da Universidade Estadual de Campinas-SP

${ }^{2}$ Professora colaboradora do Serviço de Hematologia e Hemoterapia da Faculdade de Medicina da USP-SP.

Correspondência: Márcia Torresan Delamain

Centro de Hematologia e Hemoterapia - Hemocentro - Unicamp

Rua Carlos Chagas, 480 - Cidade Universitária - CP 6198

13083-970 - Campinas, SP - Brasil

Tel. (19) 3521-8740

E-mail:marciatd@unicamp.br 
dos promissores e boa tolerabilidade. ${ }^{8}$

Para confirmar esses resultados preliminares, estudos de fases II foram desenhados para avaliar a segurança e eficácia do nilotinibe em pacientes com LMC (todas as fases da doença) resistentes e intolerantes ao imatinibe e pacientes com LLA Ph+ refratária e outras neoplasias, como mastocitose sistêmica e síndrome hipereosinofílica . A dose estabelecida para este estudo foi de $400 \mathrm{mg}$ duas vezes ao dia. Em um estudo foram inseridos 316 pacientes com LMC em fase crônica, com tempo de duração de doença de 57,3 meses. Destes pacientes, $70 \%$ eram resistentes e $30 \%$ intolerantes ao imatinibe. Estes pacientes receberam nilotinibe por 341 dias (mediana) e $15 \%$ dos pacientes tiveram a dose escalonada para $600 \mathrm{mg} .{ }^{9}{ }^{10} \mathrm{Em}$ seis meses de terapia, dos pacientes em fase crônica, $74 \%$ atingiram resposta hematológica completa (RHC), 34\% atingiram resposta citogenética completa (RCC) e $52 \%$ resposta citogenética maior (RCM). O nilotinibe foi em geral bem tolerado nos pacientes em fase crônica. Em relação aos eventos adversos hematológicos, neutropenia e plaquetopenia graus 3 e 4 ocorreram em $28 \%$ e $29 \%$ dos pacientes respectivamente, devidamente resolvida com a redução ou interrupção de dose do nilotinibe temporariamente. Os eventos adversos não-hematológicos mais freqüentes, ocorrendo em todos os graus, foram erupção cutânea, náusea, cefaléia, prurido e fadiga. Destes eventos, graus 3 e 4 ocorreram em $3 \%$ dos pacientes. O aumento de enzimas hepáticas foi a anormalidade laboratorial mais comum encontrada neste grupo de pacientes. A retenção hídrica (edema periférico e pulmonar) também foi um evento relatado pouco frequente, em cerca de $<1 \%$ dos casos graus 3 e $4 .{ }^{9}$

Um segundo grupo de pacientes neste estudo ${ }^{10} \mathrm{com}$ LMC em fase acelerada, resistentes e intolerantes ao imatinibe (119 pacientes) também foi analisado. Destes, 47\% (56 pacientes) atingiram a resposta hematológica completa, a resposta citogenética maior foi obtida por 35 pacientes $(29 \%)$ e a resposta citogenética completa em $36 \%$ dos pacientes. Os efeitos adversos hematológicos foram mais graves e freqüentes neste grupo de doentes, que apresentaram neutropenia e plaquetopenia graus 3 e 4 em $45 \%$ e $40 \%$ dos pacientes respectivamente. A retenção de fluidos com nilotinibe foi rara e em dois pacientes foi reportado edema pulmonar grau 3 e 4 . A elevação de bilirrubina e lipase grau 3 ocorreu em 9\% e 18\% dos pacientes respectivamente, resultando na descontinuação do tratamento de um paciente. ${ }^{11}$

O terceiro grupo de pacientes deste estudo formado pelos pacientes com LMC em crise blástica (CB), 120 pacientes, ou LLA $\mathrm{Ph}+(41$ pacientes) resistentes e intolerantes ao imatinibe. Os doentes em crise blástica linfóide atingiram resposta hematológica completa (7/27) ou tiveram a doença estabilizada (7/27). Dos pacientes em crise blástica mielóide, 18/87 atingiram resposta hematológica completa e 27/87 ficaram com doença estável. O nilotinibe foi relativamente bem tolerado neste grupo de doentes apesar da mielossupressão, que foi freqüentemente reportada em graus 3 e $4 .{ }^{12}$
Frente a todos estes dados e baseados nos resultados apresentados até então, podemos concluir que o nilotinibe mostrou ser um medicamento seguro, eficaz para os pacientes portadores de LMC que falharam com a terapia do imatinibe. O nilotinibe é uma droga ativa, de boa tolerabilidade e aceitação para o paciente, acarretando respostas efetivas no tratamento para LMC. Os efeitos adversos relatados pelos pacientes foram considerados de leve a moderada intensidade e de fácil resolução com a redução da dose e medidas clínicas de suporte.

\section{Dasatinibe}

$\mathrm{O}$ dasatinibe é um novo agente que foi aprovado para o tratamento de adultos com LMC em FC, FA ou crise blástica resistentes ou intolerantes ao tratamento prévio com imatinibe. Dasatinibe (Sprycel ${ }^{\circledR}$, Bristol-Myers Squibb, NY, USA) é um inibidor de tirosino quinase BCR-ABL disponível na forma oral, sendo, in vitro, 300 vezes mais ativo do que o imatinibe. $\mathrm{O}$ dasatinibe também inibe outras quinases, como a família SRC. O dasatinibe, diferente do imatinibe, inibe as formas ativa e inativa da molécula BCR-ABL. Também in vitro, o dasatinibe tem se mostrado ativo contra todas as mutações da oncoproteína BCR-ABL resistentes ao imatinibe, com exceção da T315I. ${ }^{13}$

Estudos de fase I em pacientes resistentes ou intolerantes em todas as fases da LMC, o dasatinibe levou a respostas tanto hematológicas quanto citogenéticas com uma boa tolerabilidade. Pacientes que eram intolerantes ao imatinibe não demonstraram a mesma toxicidade ao dasatinibe. Também o dasatinibe foi efetivo em todas as mutações BCR-ABL, com exceção da T315I. ${ }^{14}$

A eficácia do dasatinibe pôde ser confirmada em estudos internacionais de fase II. Foram desenhados quatro estudos abertos multicêntricos denominados START para pacientes com LMC em todas as fases, resistentes ou intolerantes ao imatinibe. A eficácia do dasatinibe, na dose de $70 \mathrm{mg}$ duas vezes por dia, pôde ser avaliada em 387 pacientes com LMC-FC (START C), 288 resistentes e 99 intolerantes ao imatinibe. A mediana de seguimento foi de 15.2 meses e as respostas duradouras (91\% com RHC e 59\% com RCM). Foi observada resposta mesmo na presença de todas as mutações BCR-ABL, com exceção da T315I. As taxas de sobrevida livre de progressão (SLP) e sobrevida global (SG) foram, respectivamente, $90 \%$ e 96\%. Em 174 pacientes com LMC-FA resistentes (161 pacientes) ou intolerantes (13 pacientes) ao imatinibe (STARTA), as RHC e RCM atingidas em uma mediana de seguimento de 14.1 meses foram, respectivamente, $45 \%$ e $39 \%$. A resposta ao tratamento não foi dependente da presença de mutações. A mediana de SLP foi de 66\%, e a SG, de $82 \%$. Em pacientes com LMC-CB mielóide (START B; $n=74$ ) ou linfóide (START L; n=42), após oito meses de seguimento, $26 \%$ e $26 \%$ atingiram RHC e $31 \%$ e $50 \%$ atingiram RCM. A mediana de duração da SLP foi de cinco meses para CB mielóide e de apenas 2.8 meses para CB linfóide. ${ }^{2}$ 
Em outro estudo de fase II, aberto, pacientes foram randomizados para dasatinibe $70 \mathrm{mg}$ duas vezes ao dia ou para um grupo controle de imatinibe $800 \mathrm{mg} /$ dia. A mudança de braço era possível em casos de falta ou perda de qualquer resposta ou intolerância. Com um seguimento de 15 meses, RHC foi atingida com dasatinibe em 93\% dos pacientes, enquanto a taxa de RHC para o grupo com imatinibe foi de $82 \%$ ( $\mathrm{p}=0.034)$. Taxas de RCM (52 vs 33\%; $\mathrm{p}=0.023)$, RCC (40 vs $16 \% ; \mathrm{p}=0.004)$ e RMM ( 16 ss $4 \% ; \mathrm{p}=0.038)$ foram significativamente mais altas no grupo do dasatinibe do que com imatinibe. A sobrevida livre de progressão foi também significativamente mais alta no grupo com dasatinibe. Entretanto, a incidência de citopenias e de derrame pleural foi maior no grupo do dasatinibe. ${ }^{2}$

As respostas de acordo com a presença de mutações BCR-ABL preexistentes foram muito parecidas nos diversos estudos. Resposta citogenética completa pode ser observada em pacientes carregando diversos tipos de mutações, com exceção da T315I . ${ }^{14}$

Toxicidade hematológica pôde ser observada mais comumente em pacientes com fase de doença mais avançada. Neutropenia e trombocitopenia graus 3 e 4 foram as citopenias mais comuns e ocorreram em cerca de $60 \%$ dos pacientes. $\mathrm{O}$ evento adverso mais comum não hematológico foi o derrame pleural, sendo responsável por uma incidência de $17 \%-27 \%$ em qualquer grau em pacientes com FC. Todos os eventos adversos puderam ser manejados clinicamente, tendo sido o dasatinibe bem tolerado em geral. ${ }^{15}$

\section{Recomendações para o uso de inibidores de tirosino quinase de segunda geração}

- Os inibidores de $2^{\circ}$ geração mostram-se eficazes e seguros no tratamento da LMC em todas as fases, nos pacientes que falharam com a terapia do imatinibe.

- Os eventos adversos relatados até então foram considerados leves a moderados e de fácil manejo com a redução de dose ou interrupção temporária da droga. A retenção hídrica (edema periférico, pleural, pulmonar) ocorreu em $<1 \%$ dos pacientes.

- Medicamentos que sabidamente interagem com as isoenzimas do citocromo $\mathrm{P} 450$ devem ser usados com cautela, devido ao risco inerente de atividade reduzida ou toxicidade exacerbada da medicação e/ou do inibidor de segunda geração.

Em relação à terapia de imatinibe, considerar:

- Resistência ao imatinibe: falha para obtenção de RHC em três meses, nenhuma resposta citogenética aos 6 meses, falha para obtenção de RCM aos 12 meses, falha para obtenção de RCC aos 18 meses.

- Intolerância ao imatinibe: pacientes que descontinuaram a terapia com imatinibe em decorrência de eventos adversos de grau 3 ou 4 e que persistem apesar de medidas de suporte ideais ou recorrência dos episódios mais de três vezes durante o tratamento; ou eventos grau 2 que persistem por mais de trinta dias.

\section{Abstract}

Despite the success with imatinib as the first choice treatment of chronic myeloid leukemia (CML), there is still a subset of patients that do not respond optimally to or are intolerant of this drug or lose response. Imatinib resistance can occur at any phase, but it is more frequent in advanced phases, with mutations in the BCR$A B L$ kinase domain being the most common mechanism of resistance. More potent tyrosine kinase inhibitors have been developed that can overcome resistance to imatinib. Nilotinib and dasatinib are good examples of new tyrosine kinase inhibitors that are available. With these new agents, patients who develop imatinib resistance or those unable to tolerate imatinib treatment can achieve significant clinical responses. Rev. bras. hematol. hemoter. 2008; 30(Supl.1):37-40.

Key words: Tyrosine kinase inhibitor; imatinib resistant; AMN107; chronic myeloid leukemia; dasatinib.

\section{Referências Bibliográficas}

1. Jabbour E, Cortes J, Ghanem H, O'Brien, Kantarjian H. Target therapy in chronic myeloid leukemia. Expert Rev. Anticancer Ther. 2008;8(1):99-110.

2. Brave M, Goodman J, Kaminskas E. Sprycel for chronic myeloid leukemia and Philadelphia chromosome-positive acute lymphoblastic leukemia resistent to or intolerant of imatinib mesylate. Clin Câncer Res. 2008;14(2):352-9.

3. Keam S. Dasatinib. In chronic myeloid leukemia and Philadelphia chromosome-positive acute lymphoblastic leukemia. Adis Drug Profile. 2008;22(1):59-69.

4. Golemovic M, Verstovsek S, Giles F, et al. AMN107, a novel aminopyrimidine inhibitor of Bcr-Abl, has in vitro activity against imatinib-resistant chronic myeloid leukemia. Clin Cancer Res. $2005 ; 11 ; 4941-7$.

5. Weisberg E, Manley PW, Breitenstein W, et al. Characterization of AMN107, a selective inhibitor of native and mutant Bcr-Abl. Cancer Cell. 2005;7:129-41.

6. Manley P, Cowan-Jacob SW, Mestran J. Advances in the structural biology, design and clinical development of Bcr-Abl kinase inhibitors for the treatment of chronic myeloid leukemia. Biochem Biophys Acta. 2005;1754:3-13.

7. Jabbour E, Corets J, O'Brien S, Giles F, Kantarjian H. New targeted therapies for chronic myelogenous leukemia: opportunities to overcome imatinib resistance. Semin Hematol. 2007; 44(Suppl 1): S25-31.

8. Le Coutre P, Baskaynak G, Tamm I, et al. Activity and induction of apoptosis of the specific tyrosine kinase inhibitor AMN 107 in bcr-abl+ cell lines and in imatinib resistant primary cells from CML patients. Blood. 2004;104:218a

9. Kantarjian HM, Giles F, Gattermann N, Bhalla K, Alimena G, Palandri F, et al. Nilotinib (formerly AMN107), a highly selective BCRABL tyrosine kinase inhibitor, is effective in patients with Philadelphia chromosome-positive chronic myelogenous leukemia in chronic phase following imatinib resistance and intolerance. Blood. 2007;110(10):3540-6. 
10. G. Rosti, Le Coutre, K. Bhalla, F. Giles, G. Ossenkoppele, A. Hochhaus, et al. Journal of Clinical Oncology, 2007 ASCO Annual Meeting Proceedings Part I. Vol 25, No.18S (June 20 Suplement) 2007: 7007

11. Le Coutre P, Ottmann OG, Giles F, Kim DW, Cortes J. Gattermann, $\mathrm{N}$, et al. Nilotinib (formerly AMN107), a highly selective BCRABL tyrosine kinase inhibitor, is active in patients with imatinibresistant or -intolerant accelerated phase chronic myelogenous leukemia. Prepublished on line Dec 2007; doi 10.1182-Blood2007-04-830196.

12. Ottmann OG, Kantarijian H, Larson, et al. A phase II study of nilotinib, a novel tyrosine kinase inhibitor administered to imatinib resistant or intolerant patients with chronic myelogenous leukemia (CML) in blast crisis (BC) or relapsed/refractory $\mathrm{Ph}+$ acute lymphoblastic leukemia (ALL). Abstract. Paper presented at American Society of Hematology48th Annual Meeting; December9-12, 2006; Orlando, Fla.

13. Hochhaus A. Dasatinib for the treatment of Philadelphia chromosome-positive chronic myelogenous leukaemia after imatinib failure. Expert Opinion. 2007;8(18):3257-64.

14. Hochhaus A. Management of Bcr-Abl-positive leukemias with dasatinib. Drug Profile. 2007;7(11):1529-36.

O tema apresentado e o convite ao autor constam da pauta elaborada pelos co-editores, Professor Ricardo Pasquini e Professor Cármino Antonio de Souza.

Avaliação: Co-editores e um revisor externo.

Publicado após revisão e concordância do editor.

Conflito de interesse: não declarado.

Recebido: $15 / 02 / 2008$

Aceito: $21 / 02 / 2008$ 